\title{
Triple ionization and frustrated triple ionization in triatomic molecules driven by intense laser fields
}

\author{
M. B. Peters $\odot,{ }^{1}$ V. P. Majety, ${ }^{2}$ and A. Emmanouilidou ${ }^{1}$ \\ ${ }^{1}$ Department of Physics and Astronomy, University College London, Gower Street, London WC1E 6BT, United Kingdom \\ ${ }^{2}$ Department of Physics and Center for Atomic, Molecular, and Optical Sciences and Technology, \\ Indian Institute of Technology Tirupati, Tirupati 517506, India
}

(Received 29 October 2020; accepted 23 March 2021; published 14 April 2021)

\begin{abstract}
We formulate a three-dimensional semiclassical model to treat three-electron escape dynamics in a strongly driven linear triatomic molecule, $\mathrm{HeH}_{2}{ }^{+}$. Our model includes the Coulomb singularities. Hence, to avoid unphysical autoionization, we employ two criteria to switch off the Coulomb repulsive force between two bound electrons and switch it on when the motion of one electron is mostly determined by the laser field. We investigate triple and so-called frustrated triple ionization. In the latter process, two electrons escape while one electron remains bound in a Rydberg state. We find that two pathways prevail in frustrated triple ionization, as in frustrated double ionization. We also find that the electron that remains in a Rydberg state is more likely to be attached to $\mathrm{He}^{2+}$ than to $\mathrm{H}^{+}$. Our results indicate that in triple and frustrated triple ionization electronic correlation is weak. Moreover, we compute the sum of the kinetic energies as well as the angular patterns of the final ion fragments in triple and frustrated triple ionization. These patterns suggest that the fragmenting molecule deviates from its initial linear configuration.
\end{abstract}

DOI: 10.1103/PhysRevA.103.043109

\section{INTRODUCTION}

Correlated multielectron escape dynamics arising in systems driven by intense infrared and midinfrared laser fields is a problem of fundamental interest. The complexity of the problem currently limits ab initio quantum mechanical computations to two-electron escape in strongly driven atoms $[1,2]$. This latter problem has also been addressed by three-dimensional quantum mechanical [3] and semiclassical techniques [4-6] that include the Coulomb singularity. Given the even larger degree of complexity, strongly driven three-electron dynamics has been addressed in few theoretical [7-10] and experimental studies [11,12]. More relevant to the current work is the classical study of driven trimers in Ref. [10] with three atoms placed far apart and each electron being bound to a different atom. The work in Ref. [10] does not address the unphysical autoionization that occurs in a classical treatment of two bound electrons when the Coulomb singularities are included. One electron can acquire a very negative energy and release energy that leads to the escape of the other bound electron. This does not occur quantum mechanically, since the energy of an electron has a lower bound. This unphysical autoionization is addressed in this semiclassical work that includes the Coulomb singularities and involves two bound electrons.

Here, we develop a three-dimensional (3D) semiclassical model to investigate three electron dynamics in strongly driven triatomic molecules. We do so in the context of the strongly driven linear molecule $\mathrm{HeH}_{2}{ }^{+}$. This model is an extension to the six-body Coulomb problem of the model we developed to describe first $\mathrm{H}_{2}[13,14]$ and then $\mathrm{D}_{3}{ }^{+}[15]$ when driven by intense laser fields. Treating unphysical autoionization is an aspect of our model introduced in this work. The latter arises since we fully account for the Coulomb singularities and in $\mathrm{HeH}_{2}{ }^{+}$more than one electron can be bound.

Using this three-dimensional (3D) semiclassical model, we account for triple and double ionization and so-called frustrated triple ionization. Frustrated ionization involves the formation of Rydberg states. Namely, an electron first tunnel ionizes in the driving laser field. Then, due to the electric field, this electron is recaptured by the parent ion in a Rydberg state [16]. In frustrated double ionization (FDI), an electron is ionized while another one remains bound in a Rydberg state at the end of the laser pulse. Frustrated double ionization accounts for roughly $10 \%$ of all ionization events. Hence, FDI is a major process in the breakup of strongly driven molecules. It has been addressed in experimental studies of $\mathrm{H}_{2}$ [17], $\mathrm{D}_{2}$ [18], and the two-electron triatomic molecules $\mathrm{D}_{3}{ }^{+}$and $\mathrm{H}_{3}{ }^{+}$ [19-21].

Two pathways account for frustrated double ionization in strongly driven two-electron diatomic and triatomic molecules [13-15]. In both pathways, one electron tunnel ionizes early on (first step), while the remaining bound electron ionizes later in time (second step). In pathway A, it is the second step that is frustrated, i.e., the initially bound electron does not escape but remains bound in a Rydberg state [13]. In pathway B, it is the first step that is frustrated, i.e., the initially tunneling electron is captured in a Rydberg state [14]. Also, electronelectron correlation, which can underlie pathway B [13,15], can be controlled with orthogonally polarized two-color linear laser fields [22,23]. Furthermore, significant enhancement of pathway B of FDI with no electronic correlation is achieved when driving triatomic molecules with counter-rotating two- 
color circular laser fields [24]. It was shown that this is due to the electron that tunnel ionizes, first hovering around the nuclei. This feature is most prevalent when 800- and 400-nm laser fields are employed with a field-strength ratio equal to two [24]. In addition, fingerprints of nuclear motion on the electron dynamics have been previously identified in frustrated double ionization $[25,26]$. Such a signature includes an oscillation in the principal $n$ quantum number [25].

In this work, we address frustrated triple ionization (FTI) where two electrons escape while one remains bound in a Rydberg state. We identify the pathways of frustrated triple ionization and compute the principle $n$ quantum number in FTI. Moreover, we compute triple as well as double ionization and discuss the role that correlation plays in the three- and two-electron escape. We also compute the distributions of the kinetic energy release and the angles of the final ion fragments in all three ionization processes.

\section{METHOD}

In the initial state of $\mathrm{HeH}_{2}{ }^{+}$, all three atoms are placed along the $z$ axis. The two hydrogen atoms are at -3.09 a.u. and -1.02 a.u., respectively, and the helium atom is at 1.04 a.u. We refer to $\mathrm{H}$ farther away from $\mathrm{He}$ as left $\mathrm{H}$ and the one closest to $\mathrm{He}$ as middle $\mathrm{H}$. We compute the distance between the two hydrogen atoms and the hydrogen and helium atoms using the quantum chemistry package MOLPRO [27]. We employ the Hartree-Fock method with the augcc-pV5Z basis set. The Hartree-Fock method overestimates by a small amount the distance between the hydrogen and the helium atoms [28]. However, we employ this method for consistency with the Hartree-Fock wave functions that we use in the potential energy terms involved in computing the exit point of the tunnel-ionizing electron [14]. The electric field is along the axis of the linear molecule, with a strength within the below-the-barrier ionization regime. As a result, one electron (electron 1) tunnel ionizes at time $t_{0}$ through the field-lowered Coulomb potential. This is a quantum-mechanical step. We employ a quantum-mechanical calculation to compute this ionization rate. Specifically, we obtain the alignment-dependent tunnel-ionization rate for $\mathrm{HeH}_{2}{ }^{+}$by employing the hybrid antisymmetrized coupled channels (haCC) method described in Refs. [29,30]. In haCC, the system is represented in a basis of neutral and singleionization channel functions. The ground state of $\mathrm{HeH}_{2}{ }^{+}$and the energetically lowest few $\mathrm{HeH}_{2}{ }^{+}$states are obtained from the quantum chemistry package Columbus [31]. A purely numerical basis is used to represent the tunneling electron, while antisymmetrization is fully enforced. Exterior complex scaling is employed in order to obtain tunnel-ionization rates. We assume that electron 1 exits along the direction of the laser field; for details on the exit point, see Ref. [14]. We compute the first ionization energy of $\mathrm{HeH}_{2}{ }^{+}$with MOLPRO and find it equal to 1.02 a.u. When the tunnel electron exits the field-lowered Coulomb barrier, we consider the momentum of the electron parallel to the field to be equal to zero. The transverse momentum is given by a Gaussian distribution. The latter arises from standard tunneling theory [32-34] and represents the Gaussian-shaped filter with an intensity-dependent width. The initially two bound electrons (electrons 2 and 3), are each represented by a microcanonical distribution for a triatomic molecule [35]. Each electron is assigned an energy equal to 2.21 a.u., which is half the ground-state energy of $\mathrm{HeH}_{2}{ }^{2+}$. Hence, in the initial state, electronic correlation is only indirectly taken into account via the energies considered in the microcanonical distributions. We initialize the nuclei at rest. Our studies suggest that an initial predissociation does not significantly alter the ionization dynamics [14].

We use an electric field of the form

$$
\overrightarrow{\mathcal{E}}(t)=\mathcal{E}_{0} \exp \left[-2 \ln 2\left(\frac{t}{\tau}\right)^{2}\right] \cos \omega_{1} t \hat{z},
$$

where $\tau=40 \mathrm{fs}$ is the full width at half maximum of the pulse duration in intensity and $\omega_{1}$ is the frequency of the laser pulse which corresponds to a wavelength of $800 \mathrm{~nm}$. The electric field strength $\mathcal{E}_{0}$ is taken equal to 0.08 a.u. We find that the threshold of the field strength for over-the-barrier ionization is equal to 0.087 a.u. We first select randomly the tunnel-ionization time $t_{0}$ in the time interval $[-2 \tau, 2 \tau]$ and we specify the initial conditions. Then, the position and momentum of the three electrons and the three nuclei are propagated classically. We do so by employing the Hamiltonian for the six-body Coulomb problem when driven by an intense laser field. We account fully with no approximation for the Coulomb forces and the interaction of each electron and nucleus with the laser field. In addition, the Coulomb singularities are explicitly included in our model; for details, see Ref. [14]. Moreover, the electron and nuclear dynamics are treated on an equal footing. We employ the Wentzel-Kramers-Brillouin approximation to allow each electron to tunnel with a quantum-mechanical probability during time propagation [13-15,25]. This description ensures that we accurately compute enhanced ionization [36-40]. Regarding enhanced ionization, when the nuclei are at a critical distance, a double-potential well is formed such that it is easier for an electron bound to the higher potential well to tunnel to the lower potential well and then ionize. A very good agreement of our previous results for $\mathrm{H}_{2}$ [13] and $\mathrm{D}_{3}{ }^{+}$[15] with experimental results $[17,20]$ justifies the approximations we consider in both the initial state and the time propagation in our model.

Here, we extend our model to treat unphysical autoionization between two bound electrons. We do so by introducing criteria to switch off the Coulomb repulsion between two bound electrons. Switching off the Coulomb repulsion has been implemented in previous work on classical calculations of electron impact on two-electron targets [41]. Here, in the initial state, we switch off the correlation between the bound electrons 2 and 3. Moreover, the Coulomb repulsion is switched on between electron 1, which tunnel ionizes in the initial state, and the other two bound electrons. During time propagation, electron 2 or 3 can quasi-ionize or ionize. When this happens, we turn on the Coulomb repulsion between this newly quasi-ionized or ionized electron and any remaining bound electrons. Moreover, if electron 1 becomes bound during time propagation, we turn off the Coulomb repulsion between electron 1 and any other bound electrons.

Hence, we must identify the time an electron ionizes or quasi-ionizes. We first define the time of ionization. In our previous work [5], the ionization time, $t_{i}^{c, 1}$, of an electron $i$ 
was defined as the time when the compensated energy $\left\{p_{x, i}^{2}+\right.$ $\left.p_{y, i}^{2}+\left[p_{z, i}-\mathcal{A}(t)\right]^{2}\right\} / 2-Z / r_{i}$ becomes positive and remains positive thereafter [42]; $\mathcal{A}(\mathrm{t})$ is the vector potential. However, in our previous studies of strongly driven two-electron atoms and molecules, the Coulomb force between the two electrons was switched on at all times. Hence, there was no need to compute the ionization time of each of the two electrons on the fly, i.e., during time propagation. This time was computed only after we have registered the events corresponding to different ionization processes, during the analysis of the trajectories. Here, for three electrons, we keep the Coulomb force between two bound electrons switched off to avoid unphysical autoionization. We employ the ionization time which is computed on the fly in order to determine when to switch on or off the Coulomb repulsion between two electrons. This time is not $t_{i}^{c, 1}$, since the latter cannot be determined during propagation. Instead, we employ the time $t_{i}^{c, 2}$ when the compensated energy of electron $i$, in addition to being positive from this time onward, also converges to its asymptotic value which is the final kinetic energy of electron $i$. We note that $t_{i}^{c, 2}>t_{i}^{c, 1}$.

However, an electron can transfer energy to the other electrons while it is not ionized. Indeed, the compensated energy of electron 1 is negative during its exit from the barrier and up until electron 1 first returns to the nuclei to transfer energy to the other electrons. In our computation, this is the case for most trajectories. During this time, we find that the motion of electron 1 along the $z$ axis is mostly influenced by the field. That is, in half the laser period $T$, the position of electron 1 along the $z$ axis has no more than one maximum. Hence, we monitor the time interval between two subsequent maxima in the position of each electron along the $z$ axis. If there is less than one maximum in a time interval $T / 2$, we register as quasi-ionization time $t_{i}^{q}$ the time at the end of this interval. Similarly, $t_{i}^{q}$ can be identified from the minima of the position of the electron along the $z$ axis.

We use the above two criteria to find when an electron is ionized, quasi-ionized, or bound. In our computations, for roughly $90 \%$ of FTI and triple and double ionization events, we find that the Coulomb repulsion between electrons 1 and $2\left(V_{12}\right)$ and between electrons 2 and $3\left(V_{23}\right)$ is never switched off. For the rest of the events, these forces are switched off and on only once. Moreover, the Coulomb repulsion between the initially bound electrons 2 and $3\left(V_{23}\right)$ is switched on and remains on only once for almost all ionization events. This switch on roughly takes place around five periods of the laser field after the start of the time propagation. We find that, in our method, at the time the $V_{23}$ potential energy is switched on, $V_{23}$ corresponds to less than $5 \%$ of the total energy. Hence, our approximation of a sudden switch on of the potential energy is very accurate.

It follows from the above that the transfer of energy from electron 1 to the other two initially bound electrons is accounted for very well. Indeed, for most events $V_{12}$ and $V_{13}$ are never turned off. If electron 2 or 3 becomes quasi-ionized or ionized during propagation, the transfer of energy from that electron to another electron is accounted for with a delay. Namely, we find that for the majority of ionization events $t_{i}^{c, 2}$ or $t_{i}^{q}$ is larger than $t_{i}^{c, 1}$ by at most $T / 2$. Hence, the $V_{23}$ potential is switched on with a delay. As a result, in our current formulation we accurately account for double ionization (DI), triple ionization (TI), and frustrated triple ionization (FTI) events. Indeed, using a reduced dimensionality quantum mechanical model, it has been previously shown that dynamical three-electron correlation is not important for double ionization [43]. Double ionization occurs when the recolliding electron and one of the two bound electrons finally escape. Hence, the findings in Ref. [43] suggest that double ionization is not affected by the electron-electron correlation between the two bound electrons, which in our model is switched on with a delay. The same holds for frustrated triple ionization. Regarding triple ionization, our formalism can still accurately describe events where energy is transferred from the recolliding electron to the other two bound electrons at the same time. This is the case since the force between the two bound electrons is eventually switched on following the transfer of energy. However, our model does not account well for triple ionization events which first involve a transfer of energy from one electron to a bound one. Then, within a very small time interval, they involve a second transfer of energy from the target electron in the first transfer of energy to another bound electron. However, we do not expect such processes to play a significant role in strongly driven $\mathrm{HeH}_{2}{ }^{+}$. In contrast, in triple ionization by single-photon absorption the two major pathways of ionization involve the transfer of energy via a sequence of two collisions that are only a few attoseconds apart. In one of the two major pathways, the target electron in the first transfer of energy, i.e., collision, becomes the impacting one in the second collision $[44,45]$.

\section{RESULTS}

Using the 3D model described above, we focus on triple ionization, frustrated triple ionization, and double ionization, where we obtained 85000,26000 , and 600000 events respectively. We find that out of all ionization events roughly $3.5 \%$ are TI events, $1 \%$ are FTI events, and $25 \%$ are DI events. In triple ionization, the resulting fragments are $\mathrm{He}^{2+}$ and two $\mathrm{H}^{+}$ions. In frustrated triple ionization, one electron stays in a Rydberg state either on $\mathrm{H}^{+}$or $\mathrm{He}^{2+}$. To identify the electrons captured in a Rydberg state with principal quantum number $n$, we first find $n_{c}=1 / \sqrt{2|\epsilon|}$, where $\epsilon$ is the final energy of the electron. Then, we assign a quantum number $n$ so that it satisfies $\left[(n-1)\left(n-\frac{1}{2}\right) n\right]^{\frac{1}{3}} \leqslant n_{c} \leqslant\left[n\left(n+\frac{1}{2}\right)(n+1)\right]^{\frac{1}{3}}$, a formula derived in Ref. [46]. However, for FTI, we find that the formation of $\mathrm{He}^{+*}$ and two $\mathrm{H}^{+}$ions is three times more likely than the formation of $\mathrm{He}^{2+}, \mathrm{H}^{*}$, and $\mathrm{H}^{+}$. Hence, in what follows we focus on the most probable channel of FTI. Moreover, we consider FTI events in high Rydberg states with $n>2$. The reason we ignore FTI events with $n=2$ is the same as for our work on $\mathrm{HeH}^{+}$[25]. Namely, an electron from the $n=1$ state of $\mathrm{H}^{+}$tunnels to the $n=2$ state of $\mathrm{He}^{2+}$. As a result, we obtain a large number of $n=2$ states. For DI, the electron that does not ionize remains bound mostly in the $n=1$ state. Moreover, for DI it is significantly more likely for the final fragments to be $\mathrm{He}^{+}$and two $\mathrm{H}^{+}$ions rather than $\mathrm{He}^{2+}, \mathrm{H}^{+}$, and $\mathrm{H}$. Hence, in what follows we focus on 

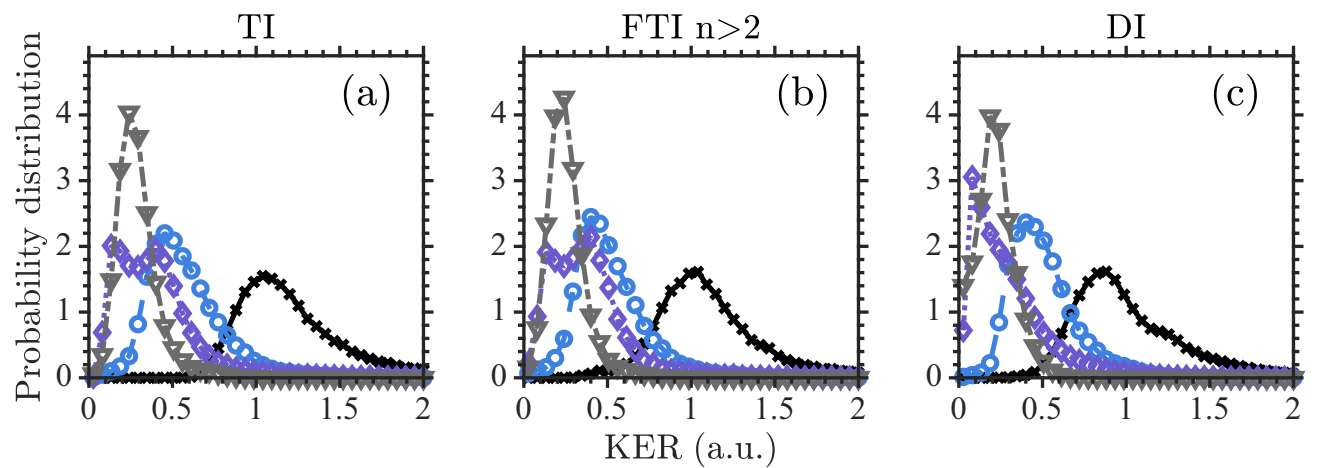

FIG. 1. Distribution of the sum of the final kinetic energies (black solid lines with crosses) of the ion fragments produced in (a) triple ionization, (b) frustrated triple ionization, and (c) double ionization. The dashed light gray lines and downward triangles depict the distribution of the final kinetic energy of the $\mathrm{He}^{2+}$ ion fragment for TI, $\mathrm{He}^{+*}$ for FTI, and $\mathrm{He}^{+}$for DI. The dashed purple lines and diamonds (dashed blue lines and circles) depict the distribution of the final kinetic energy of the middle (left) $\mathrm{H}^{+}$ion fragment for TI, FTI, and DI. All curves are normalized to one.

the most probable channel of DI as we do for FTI, unless we indicate otherwise.

\section{A. KER distributions}

In Fig. 1, we plot the kinetic energy release (KER) distributions of the final ion fragments for triple ionization, frustrated triple ionization, and double ionization. We find that the KER distribution peaks around 1 a.u. for TI and FTI, while it peaks around 0.8 a.u. for DI. These peak values are consistent with the peak values of the distributions of the internuclear distances at the time an electron tunnel ionizes last. Indeed, we find (not shown) for TI and FTI (DI) that the most probable internuclear distances are around 5 (3) a.u. between $\mathrm{He}$ and middle $\mathrm{H}$, around 7 (5) a.u. between $\mathrm{He}$ and left $\mathrm{H}$, and around 3 (3) a.u. between the two $\mathrm{H}$ atoms. Thus, the peak of the KER distribution for TI and FTI is given roughly by $2 / 7+2 / 5+1 / 3$. For DI, where the bound electron is mostly attached on $\mathrm{He}$, resulting in $\mathrm{He}^{+}$, the peak of the KER distribution is roughly given by $1 / 5+1 / 3+1 / 3$.

Also, in Fig. 1 we show that left $\mathrm{H}^{+}$is the faster fragment in all three processes. The slowest fragments are the middle $\mathrm{H}^{+}$for all three processes and $\mathrm{He}^{2+}$ for TI, $\mathrm{He}^{+*}$ for FTI, and $\mathrm{He}^{+}$for DI. This is consistent with the two Coulomb repulsive forces on the left $\mathrm{H}^{+}$ion pointing along $180^{\circ}$ with respect to the $+z$ axis. The repulsive forces on $\mathrm{He}^{2+}$ for $\mathrm{TI}, \mathrm{He}^{+*}$ for FTI, and $\mathrm{He}^{+}$for DI also add up toward $0^{\circ}$ from the $+z$ axis. However, the mass of $\mathrm{He}$ compared to $\mathrm{H}$ is four times larger. As a result, He ends up with a smaller acceleration and hence smaller final kinetic energy compared to the left $\mathrm{H}^{+}$. In addition, the repulsive forces on the middle $\mathrm{H}^{+}$from $\mathrm{He}^{2+}$ for TI, $\mathrm{He}^{+*}$ for FTI, and $\mathrm{He}^{+}$for DI and from left $\mathrm{H}^{+}$point in opposite directions. As a result, the kinetic energy of the middle $\mathrm{H}^{+}$ion is smaller compared to the left $\mathrm{H}^{+}$ion.

Moreover, we find that the KER distribution of middle $\mathrm{H}^{+}$ has a double-peak structure for TI and FTI. This double peak is associated with middle $\mathrm{H}^{+}$escaping mainly either along or at an angle with respect to the molecular axis away from $\mathrm{He}^{2+}$ for TI and $\mathrm{He}^{+*}$ for FTI. The lower (higher) peak in the KER distribution of middle $\mathrm{H}^{+}$corresponds to the middle $\mathrm{H}^{+}$escaping along (at an angle with) the molecular axis. The lower peak is more pronounced for DI, since the force on middle $\mathrm{H}^{+}$from $\mathrm{He}^{+}$in DI is smaller than the force from $\mathrm{He}^{2+}$ in TI and $\mathrm{He}^{+*}$ in FTI. As a result, the force on middle $\mathrm{H}^{+}$from $\mathrm{He}^{+}$in DI is more comparable to the force from the left $\mathrm{H}^{+}$on the middle $\mathrm{H}^{+}$. Hence, the net force on middle $\mathrm{H}^{+}$is smaller in DI compared to FTI and TI, giving rise to a smaller final kinetic energy of the middle $\mathrm{H}^{+}$for DI.

\section{B. Angular distributions}

In Fig. 2, we plot the angular distribution of the final ion fragments for triple ionization as well as for the most probable channels of frustrated triple ionization and double ionization. For TI and FTI, we find that the angular distribution of middle $\mathrm{H}^{+}$is broader compared to left $\mathrm{H}^{+}$. As discussed above, this is consistent with the Coulomb repulsive force on the middle $\mathrm{H}^{+}$ ion being smaller compared to the left $\mathrm{H}^{+}$ion. We expect that another factor contributing to the broader angular distribution of the middle $\mathrm{H}^{+}$ion is that the two electrons that tunnel ionize last mostly move between $\mathrm{He}$ and middle $\mathrm{H}^{+}$, before they both (one) escape for TI (FTI). This is due to He having a higher nuclear charge. The difference between the two angular distributions of the $\mathrm{H}^{+}$ions is even more pronounced for DI. Indeed, the full screening of $\mathrm{He}^{2+}$ by the electron bound in the $n=1$ state results in $\mathrm{He}^{+}$exerting a force toward $180^{\circ}$ on the middle $\mathrm{H}^{+}$ion that roughly cancels out the force toward $0^{\circ}$ from the left $\mathrm{H}^{+}$ion.

\section{C. $n$ quantum number for FTI}

Next, we investigate the distribution of the principal $n$ quantum number of the two main pathways of FTI. We find that both pathways $\mathrm{A}$ and $\mathrm{B}$ with $n>2$ contribute roughly the same to FTI. As already discussed, we find that formation of a Rydberg state is three times more likely for He versus $\mathrm{H}$ attachment. This is shown in Fig. 3, where we plot the distribution of the principal quantum number $n$ for pathways $\mathrm{A}$ and B of FTI. We also find that the distribution of the principal quantum number $n$ peaks around 20 when the electron remains bound in a Rydberg state of $\mathrm{He}^{2+}$ versus 10 following attachment on $\mathrm{H}^{+}$. This is expected. One assumes that the electron that tunnel ionizes last and remains bound in a Rydberg state has roughly the same energy for attachment 


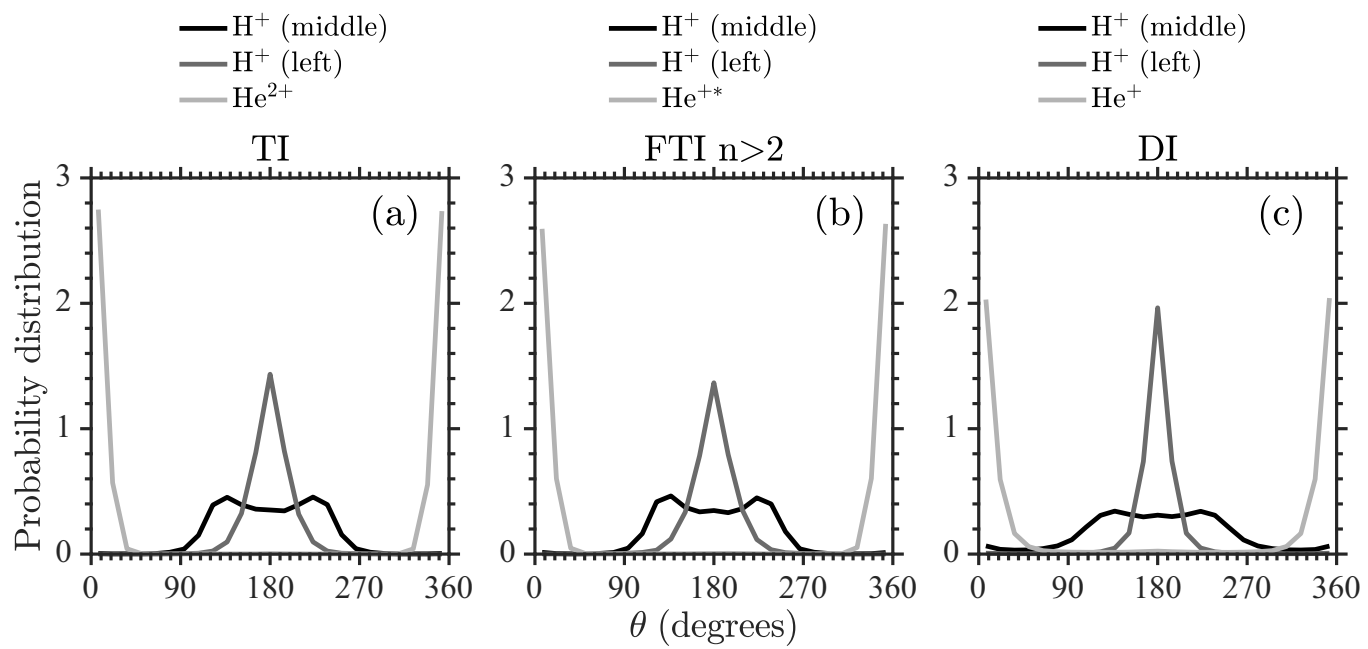

FIG. 2. Angular distributions of the ion fragments produced in (a) triple ionization, (b) frustrated triple ionization, and (c) double ionization. The escape along the $+z$ axis corresponds to $0^{\circ}$. All curves are normalized to one.

on He or H. Then, given the dependence of the energy of a hydrogenic atom on the nuclear charge $Z$ and the $n$ number, it follows that an $n$ number for attachment on $\mathrm{H}$ is equivalent to a $2 n$ number for attachment on He. Moreover, we find that the distribution of the $n$ number peaks at higher $n$ values for pathway B versus pathway A. This is consistent with the electron that remains bound in a Rydberg state in pathway $B$ being the electron that tunnel ionizes in the initial state. As a result, this electron upon its return to the nuclei has higher energy compared to the energy that an initially bound electron has when it tunnel ionizes for the last time and remains in a Rydberg state in pathway A.

\section{Correlation in triple ionization}

Our results suggest a weak effect of the correlated electron dynamics in triple ionization during fragmentation of the strongly driven triatomic molecule $\mathrm{HeH}_{2}{ }^{+}$. Specifically, we find that for roughly $20 \%$ of triple ionization events a recollision takes place where electron 1 transfers energy to both initially bound electrons 2 and 3 at the same time. For

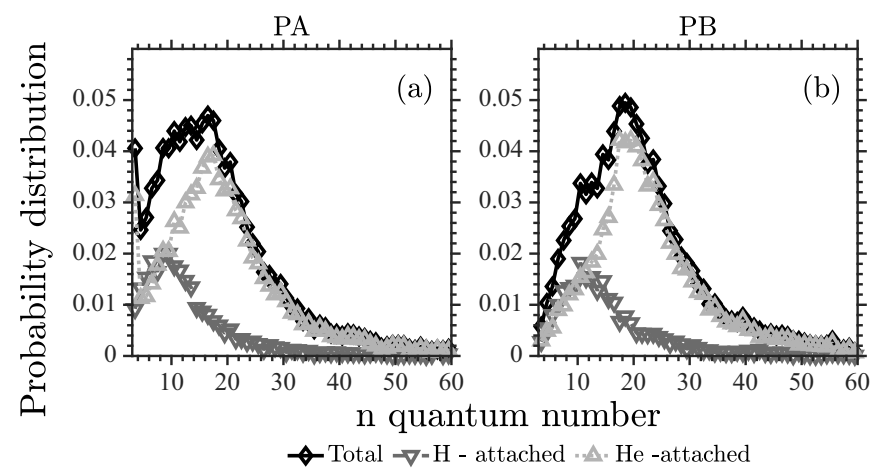

FIG. 3. Distribution of the principal $n$ quantum number for pathways A (a) and B (b) of FTI (black solid lines with diamonds). For pathways A and $\mathrm{B}$ of FTI, the distribution of the $n$ quantum number is also plotted separately when the electron remains attached to $\mathrm{He}^{2+}$ (light gray lines with upward pointing triangles) and when it remains attached on $\mathrm{H}^{+}$(dark gray lines with downward pointing triangles). these events, the distribution of the maximum of the potential energy between electrons 1 and 2 or electrons 1 and 3 as a function of time extends up to 2 a.u. (not shown). For the rest of the TI events, this maximum of the potential energy peaks overwhelmingly around very small values.

The weak electronic correlation in TI of strongly driven $\mathrm{HeH}_{2}{ }^{+}$is also supported by the distribution of the difference in ionization times of the fastest and second fastest electrons as well as the fastest and slowest electrons. These two distributions are shown in Fig. 4. We find that the electron that ionizes second has a significant probability to do so with a small time difference from the fastest one. It also has a significant probability to do so with time differences extending from one to four periods of the laser field. In contrast, the last electron to ionize does so with a distribution of time differences that roughly peaks around four periods of the laser field. This suggests that the second but mostly the last to ionize electrons escape mainly due to enhanced ionization and not due to a recollision. This is expected for molecules that are fragmenting when driven by long duration and intense pulses.

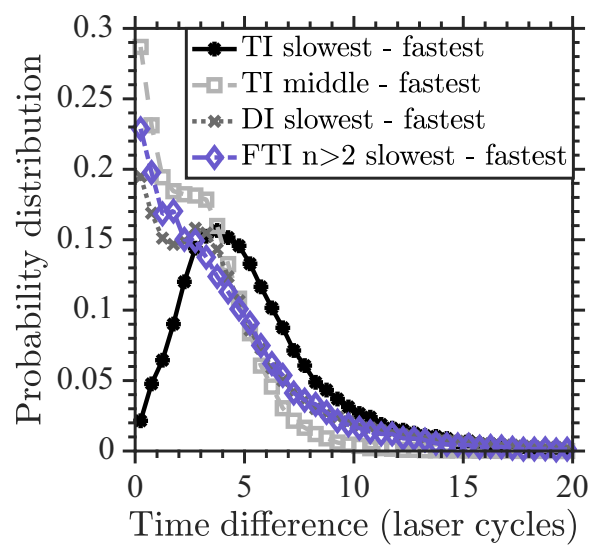

FIG. 4. Distribution of the time differences between the fastest and second fastest electrons as well as the fastest and slowest electrons in TI and between the fastest and slowest electrons in FTI and DI. 
We find this to also be the case for FTI and DI. Indeed, in Fig. 4 the distribution of the time differences between the two electrons that escape in FTI and DI extends up to roughly five periods of the laser field, suggesting that FTI and DI take place mostly due to enhanced ionization.

\section{CONCLUSIONS}

In this work, we formulate a 3D semiclassical model for a strongly driven six-body Coulomb problem to address threeelectron dynamics in strongly driven triatomic molecules. Since we include the Coulomb singularities, we address how to avoid unphysical autoionization between two bound electrons. To do so, we develop two criteria that allow us to switch off the Coulomb force between two bound electrons and switch it on when one of the two electrons ionizes or quasiionizes. In our current formulation, the two bound electrons screen each other only indirectly via their interaction with the three nuclei and the other electron. We expect that this is a very good approximation for processes involving electrons in highly excited states before the electrons actually ionize. In this case, the electrons screen each other less. Hence, we expect the current formulation to accurately describe mostly TI and FTI and less accurately DI. Indeed, in DI one electron remains bound in the $n=1$ state, resulting in higher screening of the nuclear charge during the time that it takes for the last electron to ionize.

Using this 3D semiclassical model, we address triple and double ionization as well as frustrated triple ionization in a strongly driven linear triatomic molecule, namely, $\mathrm{HeH}_{2}{ }^{+}$. We find that the electronic correlation in all three ionization processes is weak. Moreover, we find that, for frustrated double ionization, pathways A and B of FTI both contribute to the formation of Rydberg states at the end of the laser field. We also find that the electron that remains bound in FTI is roughly three times more likely to be attached to $\mathrm{He}^{2+}$ than to $\mathrm{H}^{+}$. Computing the angular distributions of the final ion fragments in all three ionization processes, we find that middle $\mathrm{H}^{+}$escapes with a broader range of angles compared to left $\mathrm{H}^{+}$and $\mathrm{He}^{2+}$ for TI, $\mathrm{He}^{+*}$ for FTI, and $\mathrm{He}^{+}$for DI. This is mainly due to the Coulomb repulsive forces on the middle $\mathrm{H}^{+}$ion from the other two ions pointing in opposite directions. Thus, the resultant force has a smaller magnitude compared to the forces on the other two ions. For DI, these opposite pointing Coulomb forces on the middle $\mathrm{H}^{+}$are even more comparable in magnitude. This is due to the screening of $\mathrm{He}^{2+}$ from the $n=1$ bound electron. As a result, the angular distribution of the middle $\mathrm{H}^{+}$ion is even broader for DI than for TI and FTI.

Our future studies will focus on generalizing our current formulation to include effective potentials that will account for the electronic repulsion between two electrons during the time that they are both bound. This will allow us to also consider processes with two bound electrons, such as frustrated double ionization.

\section{ACKNOWLEDGMENTS}

A.E. acknowledges EPSRC Grant No. EP/N031326/1. V.P.M. acknowledges support from SERB Grant No. SRG/2019/001169. The authors acknowledge the use of the UCL Myriad High Performance Computing Facility (Myriad@UCL), and associated support services, in the completion of this work.
[1] J. S. Parker, B. J. S. Doherty, K. T. Taylor, K. D. Schultz, C. I. Blaga, and L. F. DiMauro, Phys. Rev. Lett. 96, 133001 (2006).

[2] J. S. Parker, L. R. Moore, D. Dundas, and K. T. Taylor, J. Phys. B 33, L691 (2000).

[3] A. Zielinski, V. P. Majety, and A. Scrinzi, Phys. Rev. A 93, 023406 (2016).

[4] L. B. Fu, J. Liu, J. Chen, and S. G. Chen, Phys. Rev. A 63, 043416 (2001).

[5] A. Emmanouilidou, Phys. Rev. A 78, 023411 (2008).

[6] L.-B. Fu, J. Liu, and S.-G. Chen, Phys. Rev. A 65, 021406(R) (2002).

[7] Q. Tang, C. Huang, Y. Zhou, and P. Lu, Opt. Express 21, 021433 (2013).

[8] J. H. Thiede, B. Eckhardt, D. K. Efimov, J. S. PrauznerBechcicki, and J. Zakrzewski, Phys. Rev. A 98, 031401(R) (2018).

[9] K. Sacha and B. Eckhardt, Phys. Rev. A 64, 053401 (2001).

[10] Z. Q. Yuan, D. F. Ye, Y. Q. Gu, J. Liu, and L. B. Fin, Opt. Express 27, 3180 (2019).

[11] R. Moshammer, B. Feuerstein, W. Schmitt, A. Dorn, C. D. Schröter, J. Ullrich, H. Rottke, C. Trump, M. Wittmann, G. Korn, K. Hoffmann, and W. Sandner, Phys. Rev. Lett. 84, 447 (2000).

[12] K. Zrost, A. Rudenko, T. Ergler, B. Feuerstein, V. L. B. de Jesus, C. D. Schröter, R. Moshammer, and J. Ullrich, J. Phys. B 39, S371 (2006).
[13] A. Emmanouilidou, C. Lazarou, A. Staudte, and U. Eichmann, Phys. Rev. A 85, 011402(R) (2012).

[14] H. Price, C. Lazarou, and A. Emmanouilidou, Phys. Rev. A 90, 053419 (2014).

[15] A. Chen, H. Price, A. Staudte, and A. Emmanouilidou, Phys. Rev. A 94, 043408 (2016).

[16] T. Nubbemeyer, K. Gorling, A. Saenz, U. Eichmann, and W. Sandner, Phys. Rev. Lett. 101, 233001 (2008).

[17] B. Manschwetus, T. Nubbemeyer, K. Gorling, G. Steinmeyer, U. Eichmann, H. Rottke, and W. Sandner, Phys. Rev. Lett. 102, 113002 (2009).

[18] W. Zhang, H. Li, X. Gong, P. Lu, Q. Song, Q. Ji, K. Lin, J. Ma, H. Li, F. Sun, J. Qiang, H. Zeng, and J. Wu, Phys. Rev. A 98, 013419 (2018).

[19] J. McKenna, A. M. Sayler, B. Gaire, N. G. Johnson, K. D. Carnes, B. D. Esry, and I. Ben-Itzhak, Phys. Rev. Lett. 103, 103004 (2009).

[20] J. McKenna, A. M. Sayler, B. Gaire, N. G. Kling, B. D. Esry, K. D. Carnes, and I. Ben-Itzhak, New J. Phys. 14, 103029 (2012).

[21] A. M. Sayler, J. McKenna, B. Gaire, N. G. Kling, K. D. Carnes, and I. Ben-Itzhak, Phys. Rev. A 86, 033425 (2012).

[22] A. Chen, M. F. Kling, and A. Emmanouilidou, Phys. Rev. A 96, 033404 (2017).

[23] S. Larimian, C. Lemell, V. Stummer, J.-W. Geng, S. Roither, D. Kartashov, L. Zhang, M.-X. Wang, Q. Gong, L.-Y. Peng, 
S. Yoshida, J. Burgdörfer, A. Baltuska, M. Kitzler, and X. Xie, Phys. Rev. A 96, 021403(R) (2017).

[24] G. P. Katsoulis, R. Sarkar, and A. Emmanouilidou, Phys. Rev. A 101, 033403 (2020).

[25] A. Vila, Z. Jinzhen, A. Scrinzi, and A. Emmanouilidou, J. Phys. B 51, 065602 (2018).

[26] W. Zhang, X. Gong, H. Li, P. Lu, F. Sun, Q. Ji, K. Lin, J. Ma, H. Li, J. Qiang, F. He, and J. Wu, Nat. Commun. 10, 757 (2019).

[27] H.-J. Werner, P. J. Knowles, G. Knizia, F. R. Manby, M. Schütz, P. Celani, W. Györffy, D. Kats, T. Korona, R. Lindh et al., MOLPRO, version 2018.1, a package of ab initio programs, https: //www.molpro.net.

[28] P. Palmieri, C. Puzzarini, V. Aquilanti, G. Capecchi, S. Cavalli, D. De Fazio, A. Aguilar, X. Gimenez, and J. M. Lucas, Mol. Phys. 98, 1835 (2000).

[29] V. P. Majety, A. Zielinski, and A. Scrinzi, New J. Phys. 17, 063002 (2015).

[30] V. P. Majety and A. Scrinzi, J. Phys. B: At. Mol. Opt. Phys. 48, 245603 (2015).

[31] H. Lischka, T. Müller, P. G. Szalay, I. Shavitt, R. M. Pitzer, and R. Shepard, WIREs Comput. Mol. Sci. 1, 191 (2011).

[32] N. B. Delone and V. P. Krainov, J. Opt. Soc. Am. B 8, 1207 (1991).

[33] N. B. Delone and V. P. Krainov, Phys. Usp. 41, 469 (1998).
[34] L. Fechner, N. Camus, J. Ullrich, T. Pfeifer, and R. Moshammer, Phys. Rev. Lett. 112, 213001 (2014).

[35] A. Chen, C. Lazarou, H. Price, and A. Emmanouilidou, J. Phys. B 49, 235001 (2016).

[36] H. Niikura, F. Légaré, R. Hasbani, A. D. Bandrauk, M. Y. Ivanov, D. M. Villeneuve, and P. B. Corkum, Nature (London) 417, 917 (2002).

[37] T. Zuo and A. D. Bandrauk, Phys. Rev. A 52, R2511(R) (1995).

[38] T. Seideman, M. Y. Ivanov, and P. B. Corkum, Phys. Rev. Lett. 75, 2819 (1995).

[39] D. M. Villeneuve, M. Y. Ivanov, and P. B. Corkum, Phys. Rev. A 54, 736 (1996).

[40] E. Dehghanian, A. D. Bandrauk, and G. L. Kamta, Phys. Rev. A 81, 061403(R) (2010).

[41] D. R. Schultz, L. Meng, and R. E. Olson, J. Phys. B 25, 4601 (1992).

[42] J. G. Leopold and I. C. Percival, J. Phys. B 12, 709 (1979).

[43] D. K. Efimov, J. S. Prauzner-Bechcicki, and J. Zakrzewski, Phys. Rev. A 101, 063402 (2020).

[44] A. Emmanouilidou and J. M. Rost, J. Phys. B 39, 20 (2006).

[45] J. Colgan, A. Emmanouilidou, and M. S. Pindzola, Phys. Rev. Lett. 110, 063001 (2013).

[46] R. L. Becker and A. D. MacKeller, J. Phys. B 17, 3923 (1984). 